\title{
Multiplexing and Error Control Scheme with Modified Hybrid ARQ for Body Area Network employing IEEE 802.15.6 in UWB-PHY
}

\author{
Kento Takabayashi* \\ Graduate School of \\ Engineering, Yokohama \\ National University \\ 79-5 Tokiwadai, Hodogaya, \\ Yokohama, 240-8501, Japan \\ takabayashi@kohnolab. \\ dnj.ynu.ac.jp
}

\author{
Hirokazu Tanaka ${ }^{\dagger}$ \\ Graduate School of \\ Engineering, Yokohama \\ National University \\ 79-5 Tokiwadai, Hodogaya, \\ Yokohama, 240-8501, Japan \\ hi.tanaka@m.ieice.org
}

\author{
Ryuji Kohno \\ Graduate School of \\ Engineering, Yokohama \\ National University \\ 79-5 Tokiwadai, Hodogaya, \\ Yokohama, 240-8501, Japan \\ kohno@ynu.ac.jp
}

\author{
Chika Sugimoto \\ Graduate School of \\ Engineering, Yokohama \\ National University \\ 79-5 Tokiwadai, Hodogaya, \\ Yokohama, 240-8501, Japan \\ chikas@ynu.ac.jp
}

\begin{abstract}
Recently, wireless body area networks (WBAN) have attracted attention. As an international standard of WBANs suitable for medical fields, IEEE802.15.6 has been established in February 2012. In this standard, it is possible to divide the quality of service (QoS) of handled data into 7 different user priorities. However, means for the concrete realization in accordance with these priorities is implementationdependent in UWB-PHY. From this problem, we has employed Decomposable codes and Weldon's ARQ for the proposed scheme in order to adjust balance between error correcting capability of codes and the number of retransmission according to each medical QoS. We have showed that the proposed scheme has been able to improve BER and throughput performances satisfying each medical QoS by computer simulation.
\end{abstract}

${ }^{*}$ This auther is a graduate student. His research interests in NGN such as Wireless Body Area Network, coding theory in wireless communications and ultra-wideband communications.

$\dagger_{\text {This }}$ auther also belong to TOSHIBA CORPORATION and University of Oulu Research Institute Japan - CWCNippon

Yokohama Mitsui Bldg. 15F, 1-1-2 Takashima, Nishi, Yokohama, 220-0011, Japan

Permission to make digital or hard copies of all or part of this work for personal or classroom use is granted without fee provided that copies are not made or distributed for profit or commercial advantage and that copies bear this notice and the full citation on the first page. To copy otherwise, to republish, to post on servers or to redistribute to lists, requires prior specific permission and/or a fee.

UWBAN-2013 2013, September 30-October 02

Copyright $\odot 2013$ ICST 978-1-936968-89-3

DOI 10.4108/icst.bodynets.2013.253574

\section{Keywords}

Hybrid ARQ, IEEE802.15.6, QoS parameter, Decomposable codes, UWB

\section{INTRODUCTION}

Recently, wireless body area networks (WBAN) have attracted attention [1]-[6]. As an international standard of WBANs suitable for medical fields, IEEE802.15.6 has been established in February 2012. IEEE802.15.6 has been defined to be able to deal with a variety of information around the human body such as biological information. Additionally, in this standard, it is possible to divide the quality of service (QoS) of handled data into 7 different user priorities [8]. However, means for the concrete realization in accordance with these priorities is implementation-dependent. Besides, error control methods have not been prescribed so that error detecting and correcting capabilities and permissible delay can be optimized for each of them.

Two modes, Default mode and High QoS mode, are just defined in UWB physical layer in IEEE802.15.6 assumed in this study as follows. We have summarized modulation, encoding, and use of Hybrid ARQ for each mode in Table 1.

Table 1: Modulation, encoding, and use of Hybrid ARQ for each mode in UWB physical layer

\begin{tabular}{|c|c|c|}
\hline & Default mode & High QoS mode \\
\hline Modulation & $\begin{array}{c}\text { Mandatory: } \\
\text { on-off keying } \\
\text { Option: DPSK }\end{array}$ & $\begin{array}{c}\text { Mandatory: DPSK } \\
\text { Option: } \\
\text { on-off keying }\end{array}$ \\
\hline FEC & $(63,51)$ & $(126,63)$ shortened \\
BCH code & BCH code \\
\hline Hybrid ARQ & not use & use \\
\hline
\end{tabular}


- Hybrid ARQ is used only in High QoS mode, and supplementary error control in Default mode is not enough.

- Even though user priority is divided into seven levels, each parameter for the QoS parameter is not assigned in UWB-PHY.

From these problems, we also incorporate Hybrid ARQ in Default mode and can vary how to use ARQ or FEC by QoS parameters. And yet, only the change of each parameter makes it possible for us to receive the data even if the data with different user priorities are mixed.

In this study, we have examined the implementation techniques for QoS according to the priority and evaluated the performance by computer simulation. Specifically, we has employed Decomposable codes [13] and Weldon's ARQ [9] for the proposed scheme in order to adjust balance between error-correcting capability of codes and the number of retransmission according to each medical QoS. Numerical results have illustrated the proposed scheme has been able to improve BER and throughput performances satisfying each medical QoS.

\section{SYSTEM MODEL}

In this study, we assume the following use case.

Namely, suppose data 1 that had been accumulated and data 2 are transmitted together to a WBAN coordinator. In this situation, the user priority of data 2 is 6 and that of data 1 is 5 . The user priority has followed the same as in the reference [8].

As a wireless communication system, UWB is used in this study. UWB is suitable for such systems because of its characteristics such as high-speed transmission, being shared with existing wireless communication systems and so on.

Figure 1 shows the supposed use case. Previously, you had acquired one biological information in single device. However, in this study, these data are got and multiplexed by a device which can get two or more vital data such as "Silmee" [17].

The overall system configuration is shown in Figure 2. A multiplexing part of the system is represented in Figure 3. First, each data is added to the user priority. Second, add each header with the user priority to these data. Then, these data are parameters for ARQ and encoded are decided according to the priority. Finally, each data with different user priority is multiplexed.

In UWB-PHY, this multiplexed data is modulated by DPSK or PPM. Then, Direct Sequence Spread Spectrum (DSSS) is applied because of mitigating strong multipass or multi-user interference by other UWB devices.

At the receiving end, the reverse operation is done at the transmitter. If errors aren't detected, ACK is transmitted to the transmitter. In the case that the transmitter can receive ACK, next data is send. However, if errors are detected, NACK is transmitted. The previous data is retransmitted

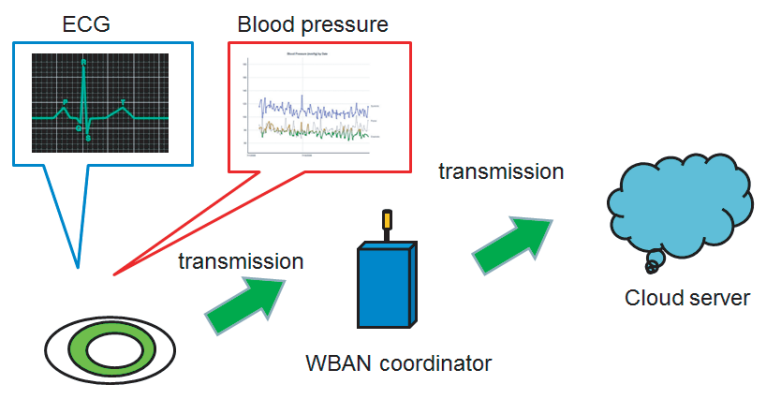

WBAN device

Figure 1: Supposed use case

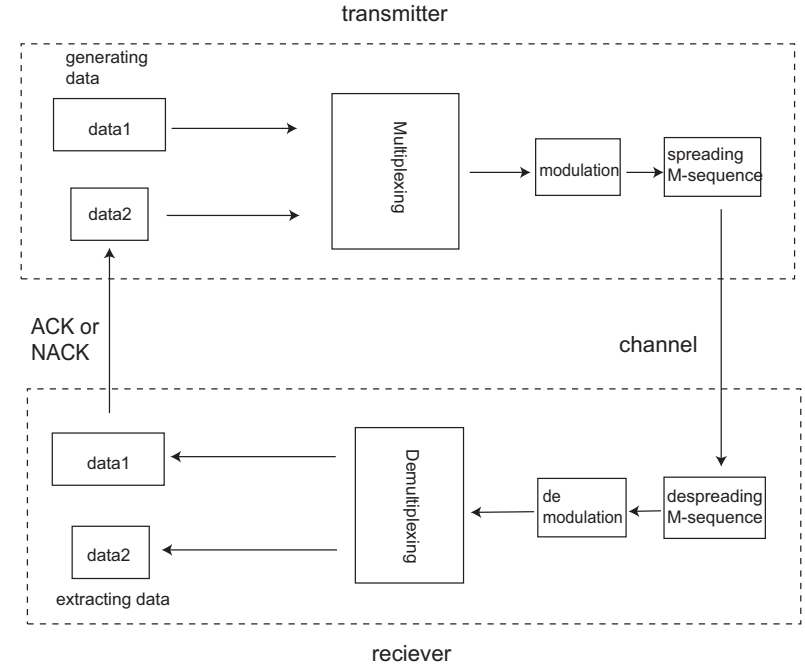

Figure 2: Overall view of the system in the proposed scheme

in the case that NACK is received or the transmitter cannot receive ACK or NACK.

In the study, the multiplexing scheme is an important point. In the next section, we will explain the details of this scheme.

\section{PROPOSED SCHEME}

\subsection{Modification in the proposed scheme}

Firstly, in the proposed scheme, the following two points are modified.

- we apply Weldon's ARQ [9] rather than selective-repeat ARQ which is a conventional ARQ protocol. This is a retransmission method: A method cascades several copies of the original data each retransmission and determined to be a successful transmission if any of them is sent correctly.

- Another is a method with Decomposable codes [13]. Decomposable codes are in appearance $(\mathrm{n}, \mathrm{k})$ linear 


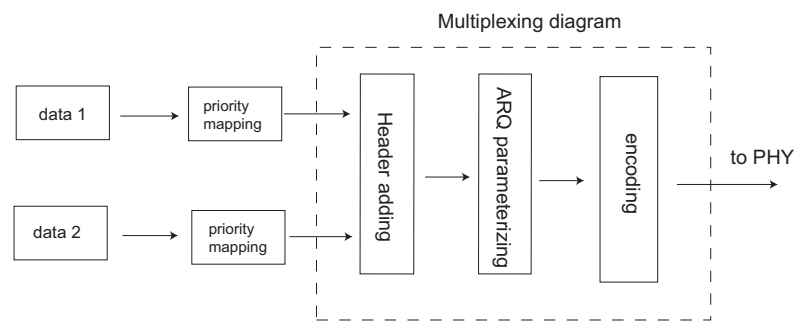

Figure 3: Block diagram of the proposed multiplexing scheme

block codes $C$, but those have $m$ element codes, $C_{1}^{\prime}, C_{2}^{\prime}$, $\ldots, C_{m}^{\prime}$, whose code length is equal to that of other element codes and shorter than that of $C$, and then structured by direct sum of what these element codes are replaced with. By using these codes, we can structure error-correcting code with higher error correcting capability by cascading several data encoded in the same FEC. In references [14] and [15], examples of Decomposable codes are introduced.

The reason these methods are used is that Decomposable codes can be used even after the third retransmission, while the FEC defined by IEEE802.15.6 is back to the first retransmission at the end of the second retransmission and Weldon's ARQ can be tailored to the characteristic of Decomposable codes or user priority unlike selective-repeat ARQ because of adjustment of the number of data copies.

Table 2 summarizes the difference between the conventional scheme and the proposed one. The top left is the conventional scheme and the bottom right is the proposed one in the table.

Secondly, in this proposed scheme, blood pressure and ECG data are not obtained by each measuring device. Instead, those data got and multiplexed by single device which can get two or more vital information. The multiplexing scheme is shown in Figure 4. In the MAC layer, each user priority is determined and parameters of Weldon's ARQ and Decomposable codes are set accordingly. After that, these two data are multiplexed in the PHY.

We detail a specific example in the following sections.

\subsection{Weldon's ARQ}

This section describes a retransmission method that E.J.Weldon proposed [9]. Retransmission is performed by the following procedure.

1. If the ACK is received, then send a new block.

2. If NACK is received after the transmission of a block, the block is repeated $n_{1}$ times.

3. If all $n_{1}$ copies are incorrect, then transmits the block $n_{2}$ times.

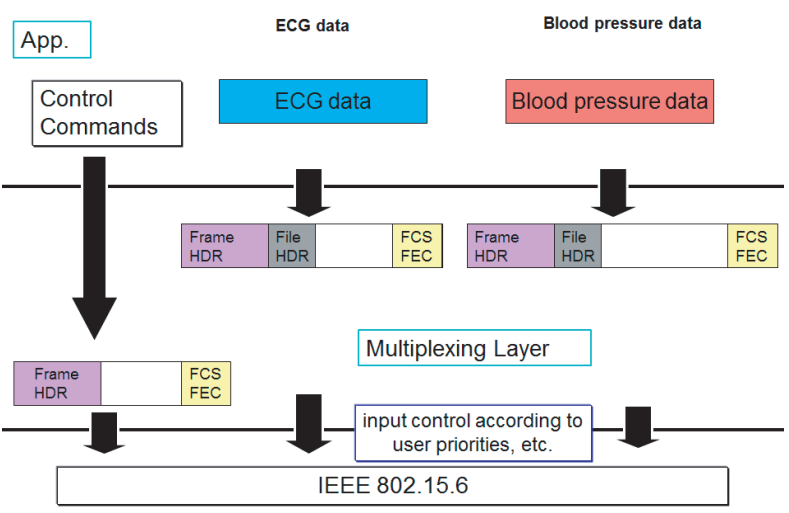

Figure 4: Multiplexing scheme in the proposal

4. After repeating the same operation $n_{3}, n_{4}, \ldots$ times, if all $n_{q-1}$ copies are in error, the block is repeated $n_{q}$ times and buffer overflow occurs.

5. If all $n_{q}$ copies are in error, the block is repeated $n_{q}$ times again and buffer overflow occurs. At this time, the block is repeated $n_{q}$ times until the transmission is successful.

Note that in the case $q=1, n_{1}=1$, it would just be selective-repeat ARQ.

\subsection{Procedure of the proposed scheme}

As Decomposable code, punctured convolutional codes [11] whose constraint length $K$ is 3 and code rate $R$ equals $7 / 8$, $5 / 6,3 / 4$ and $1 / 2$ are used. The reason is that the punctured convolutional code is generated based on the convolutional code whose generator polynomial is $[7,5]$ and code rate $R=1 / 2$. Thus, the codeword with $R=1 / 2$ can be configured by combining codeword 1 with $R=7 / 8$ generated like Figure 5 and some subcodewords of codeword 1' in Figure 6 in sequence. The correction capability is becoming higher gradually because the code rate increases in the order $7 / 8,5 / 6,3 / 4$ and $1 / 2$. This is considered that these codewords and subcodewords are elements of the original codeword with $R=1 / 2$. Then, these are just Decomposable codes.

\subsection{Procedure of the proposed scheme}

In the proposed method, retransmission is carried out as follows:

1. First, information $m$ is encoded by the punctured convolutional code and codeword 1 is transmitted. If errors aren't detected, next information is sent.

2. If errors are detected, then buffer the transmitted codeword 1 and repeat first subcodeword of codeword 1' $n_{1}$ times.

3. Sent subcodeword and buffered codeword are combined and reconstructed codeword is decoded. 
Table 2: Summary of the difference between the conventional scheme and the proposed one

\begin{tabular}{|l|l|l|}
\hline & Selective-Repeat ARQ & Weldon's ARQ \\
\hline $\begin{array}{l}\text { Hybrid ARQ-IR } \\
\text { IEEE defined in }\end{array}$ & $\begin{array}{l}\text { "conventional scheme" } \\
\text { back to the first } \\
\text { retransmission at the end of } \\
\text { the second retransmission }\end{array}$ & $\begin{array}{l}\text { The error correcting code } \\
\text { defined in IEEE802.15.6 can } \\
\text { be only configured }\end{array}$ \\
\hline Decomposable codes & $\begin{array}{l}\text { We must configure } \\
\text { Decomposable codes by } \\
\text { retransmitting data one by one }\end{array}$ & $\begin{array}{l}\text { "proposed scheme" } \\
\text { We can use those even after the third } \\
\text { retransmission and tailor those } \\
\text { to the characteristics }\end{array}$ \\
\hline
\end{tabular}

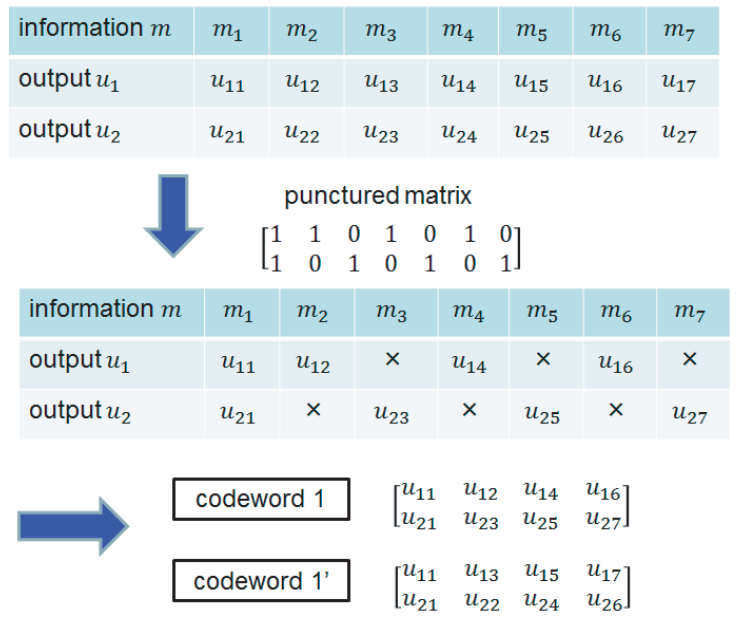

Figure 5: A method of generating the punctured convolutional code

4. If all $n_{1}$ copies are incorrect, then buffer one of the reconstructed codeword and repeat next subcodeword $n_{2}$ times.

5. Repeat until errors are not detected or you send all subcodewords.

6. If all $n_{k-1}$ copies are incorrect after sending all subcodewords, then buffer only codeword 1 and repeat codeword 1' $n_{k}$ times.

7. Codeword 1' and buffered codeword 1 are combined and original codeword with $R=1 / 2$ is decoded. If all $n_{k}$ copies are incorrect, then buffer one of the codeword 1 ' repeated $n_{k}$ times and transmits the codeword 1 $n_{k+1}$ times.

8. Repeat until no errors or the maximum number of retransmissions, $q$.

Figure 7 shows the flowchart of the proposed scheme.

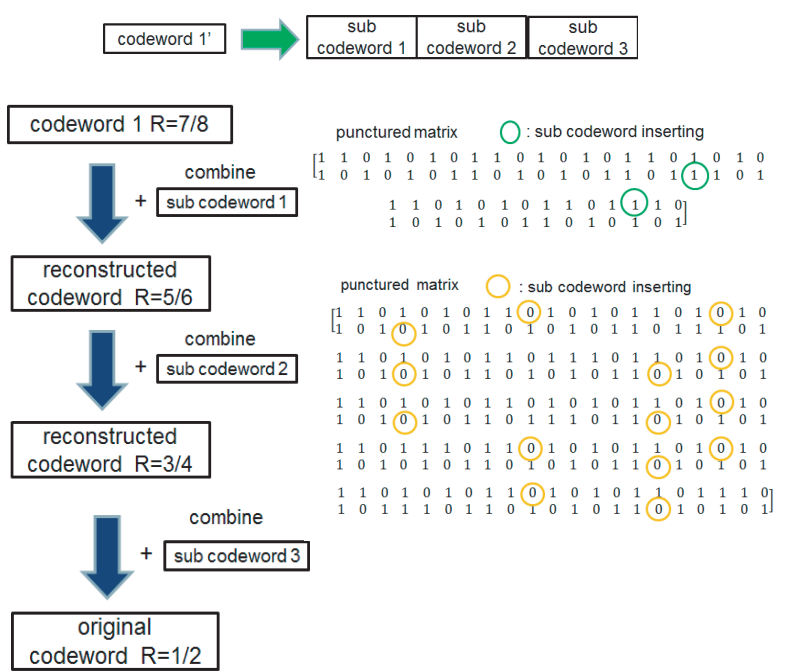

Figure 6: A method of reconstructing punctured convolutional codes

\section{COMPUTER SIMULATION}

\subsection{Supposed model and parameters of com- puter simulation}

This chapter shows computer simulations about the proposed scheme.

In the supposed use case, handling of each data is determined as shown in Table 3, 4 and ??. With respect to blood pressure with low user priority, the number of copies, $n_{i}$, and the maximum number of retransmissions, $q$, of Weldon's ARQ are large because a certain amount of latency is allowed and the residual BER and throughput performance may be improved slowly. On the other hand, in relation to ECG, there is a need for real time property because of the higher user priority. Hence we want to reduce latency and improve the throughput performance. So, $n_{i}$ and $q$ are small.

Block configuration is represented by Figure 8 .

Suppose channel model is AWGN. We make reference to [16] about data rate of vital data. Simulation parameters are summarized in Table 5. 


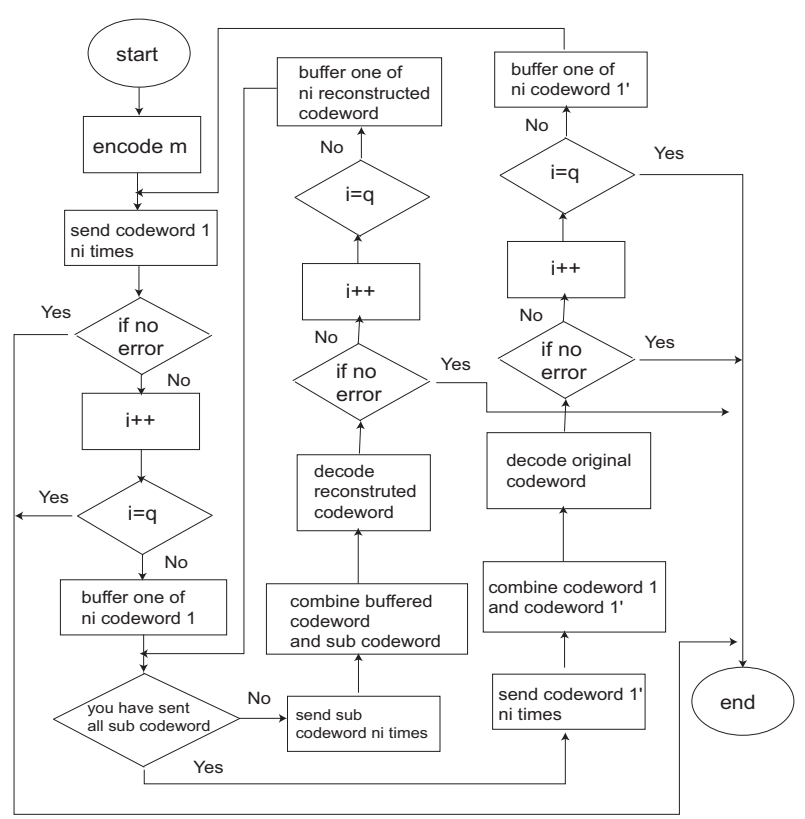

Figure 7: The flowchart of the proposed scheme

Table 3: Handling of each data in the supposed situation

\begin{tabular}{|c|c|c|}
\hline Data Types & Blood pressure & ECG \\
\hline user priority & 5 & 6 \\
\hline Latency & allowed to some extent & small \\
\hline $\begin{array}{c}\text { The maximum number of } \\
\text { retransmissions, } q\end{array}$ & 10 & 5 \\
\hline
\end{tabular}

\subsection{Results}

Figure 9 and 10 show the residual BER and throughput performance in the proposed method in AWGN for $E_{b} / N_{0}$. About the residual BER performance, that of blood pressure has been improved better than ECG, while the throughput performance of ECG has been improved better than blood pressure. The reason is that about blood pressure, the correction capability is higher because the $n_{i}$ and $q$ are large, whereas the throughput greatly decreases by the large $n_{i}$, and about ECG, the reverse can be considered. In addition, the proposal has better performance than the conventional scheme, because $n_{i}$ and $q$ are varied according to each QoS and Decomposable codes with various coding rates are used.

Figure 11 shows the residual BER and throughput performance for latency in the proposed method in AWGN and

Table 4: The number of copies $n_{i}$ for each data in AWGN

\begin{tabular}{|c|c|c|c|c|c|c|c|c|c|c|c|}
\hline$i$ & 0 & 1 & 2 & 3 & 4 & 5 & 6 & 7 & 8 & 9 & 10 \\
\hline Blood pressure, $n_{i}$ & 1 & 5 & 6 & 7 & 8 & 8 & 8 & 8 & 8 & 8 & 8 \\
\hline ECG, $n_{i}$ & 1 & 1 & 1 & 1 & 2 & 3 & - & - & - & - & - \\
\hline
\end{tabular}

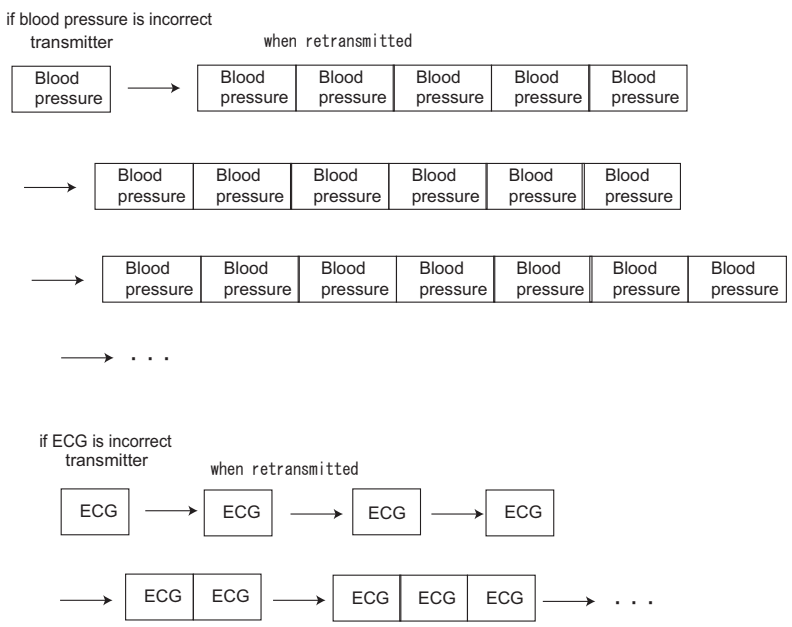

Figure 8: An example of blocks in the supposed situation

Table 5: Simulation parameters

\begin{tabular}{|c|c|}
\hline Channel model & AWGN \\
\hline Pulse shape & modulated gauspulse \\
\hline Bandwidth & $500 \mathrm{MHz}$ \\
\hline Modulation & DBPSK \\
\hline FEC & $\begin{array}{l}\mathrm{R}=7 / 8,5 / 6,3 / 4 \text { and } 1 / 2 \\
\mathrm{~K}=3 \text { convolutional codes }\end{array}$ \\
\hline ARQ protocol & Weldon's ARQ \\
\hline Spreading sequence & M-sequence \\
\hline Spreading Factor & 7 \\
\hline Message length & 756 bits \\
(Blood pressure) & $(252$ bits) \\
(ECG) & $(504$ bits) \\
\hline Data rate & $2.4 \mathrm{kbps}$ \\
\hline $\begin{array}{c}\text { Total number of bits } \\
\text { (Blood pressure) } \\
\text { (ECG) }\end{array}$ & $15.12 \mathrm{Mbits}$ \\
$(5.04 \mathrm{Mbits})$ \\
$(10.08 \mathrm{Mbits})$
\end{tabular}

in the case $E_{b} / N_{0}=5 d B$. Latency has become larger, the residual BER performance of blood pressure has improved more. On the one hand, the throughput of that have gradually improved because of its large $n_{i}$. With regard to ECG, the larger throughput has been obtained with little latency by its small $n_{i}$.

From these figures, the proposed method satisfies requirements of QoS of blood pressure and ECG. Hence, regarding blood pressure, a delay is acceptable because of its low user priority and the residual BER performance has been improved reliably, while about ECG with high user priority the real time property can be ensured and the throughput performance has been improved certainly. On the other hand, it can also be seen that the conventional scheme, especially Default mode, dose not satisfie each QoS. The reason is that Default mode does not use ARQ and FEC with low coding rate.

\section{CONCLUSION}




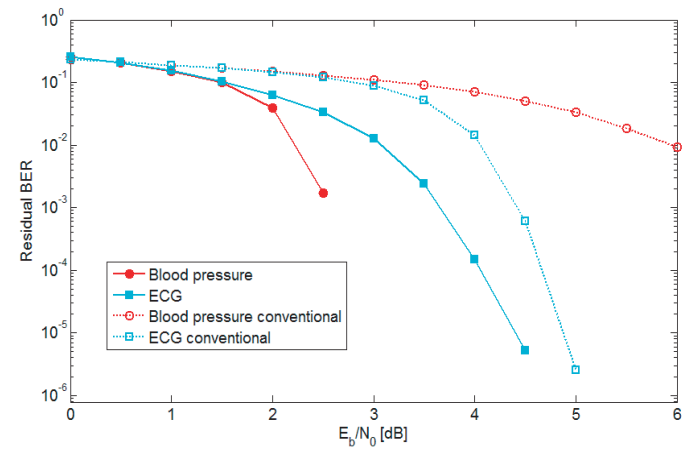

Figure 9: Residual BER performance in the proposed method in AWGN for $E_{b} / N_{0}$

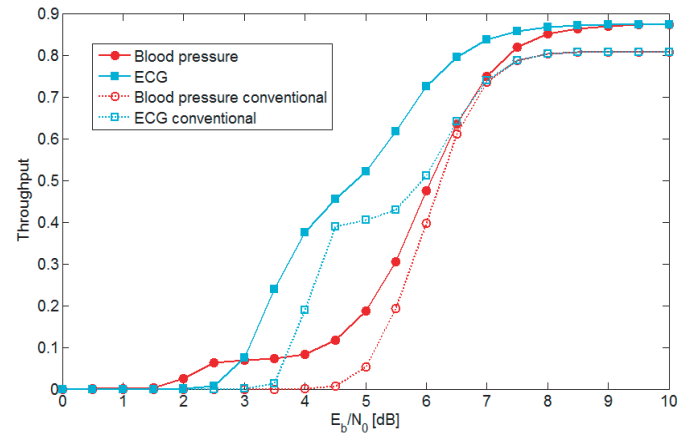

Figure 10: The throughput performance in the proposed method in AWGN for $E_{b} / N_{0}$

In this study, we have presented a use case about QoS for medical applications in IEEE802.15.6 . In particular, we has employed Decomposable codes and Weldon's ARQ for the proposed scheme in order to adjust balance between errorcorrecting capability of codes and the number of retransmission according to each medical QoS. Then we have showed that the proposed scheme has been able to improve BER and throughput performances satisfying each medical QoS by computer simulation.

In the future, we will consider a more realistic use case like referrence [7] or [12]. In additon, Decomposable codes and parameters of Weldon's ARQ will be optimized. Finally, we will analyze the scheme theoretically.

\section{REFERENCES}

[1] Huasong Cao, Victor Leung, Cupid Chow, Henry Chan, "Enabling technologies for wireless body area networks: A survey and outlook," Communications Magazine, IEEE, Volume: 47, Issue: 12, pp.84-93, Dec. 2009

[2] Hariharasudhan Viswanathan, Baozhi Chen, and Dario Pompili, "Research challenges in computation, communication, and context awareness for ubiquitous healthcare," Communications Magazine, IEEE,

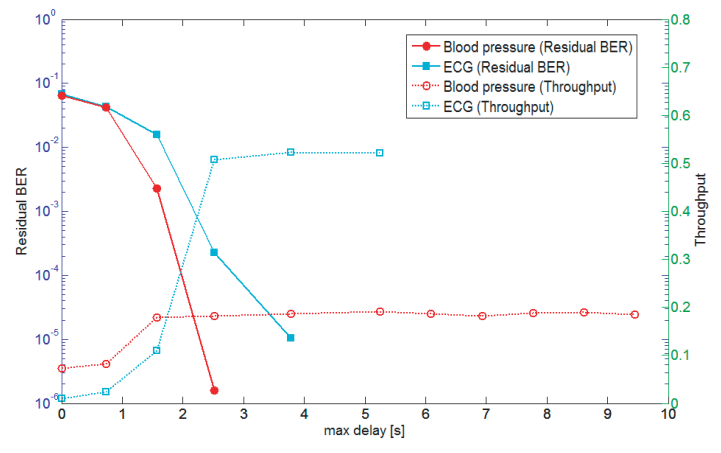

Figure 11: The throughput and residual BER performance for latency in the proposed method in $5 \mathrm{~dB}$

Volume: 50, Issue: 5, pp.92-99, May 2012

[3] Christian Bachmann, Maryam Ashouei, Valer Pop, Maja Vidojkovic, Harmke de Groot, and Bert Gyselinckx, "Low-power wireless sensor nodes for ubiquitous long-term biomedical signal monitoring," Communications Magazine, IEEE, Volume: 50, Issue: 1, pp.20-27, Jan. 2012

[4] João M. L. P. Caldeira and Joel J. P. C. Rodrigues, "Toward ubiquitous mobility solutions for body sensor networks on healthcare," Communications Magazine, IEEE, Volume: 50, Issue: 5, pp.108-115, May 2012

[5] Athanassios Boulis, David Smith, Dino Miniutti, Lavy Libman and Yuriy Tselishchev, "Challenges in body area networks for healthcare: the MAC," Communications Magazine, IEEE, Volume: 50, Issue: 5, pp.100-106, May 2012

[6] Alan Wong, Mark Dawkins, Gabriele Devita, Nick Kasparidis, Andreas Katsiamis, Oliver King, Franco Lauria, Johannes Schiff, Alison Burdett, "A 1V 5mA multimode IEEE 802.15.6/bluetooth low-energy WBAN transceiver for biotelemetry applications," Solid-State Circuits Conference Digest of Technical Papers (ISSCC), 2012 IEEE International, 300-302, Feb 2012

[7] A. Taparugssanagorn, C. Pomalaza-Ráez, A. Isola, R. Tesi, M. Hämäläinen, J. Iinatti, "UWB Channel Modelling for Wireless Body Area Networks in a Hospital," International Journal of Ultra Wideband Communications and Systems, Vol.1, No.4, pp.226-236, 2010

[8] IEEE Standard for Information technology Telecommunications and information exchange between systems - Local and metropolitan area networks- Specific requirements, "Part 15.6: Wireless Medium Access Control (MAC) and Physical Layer (PHY) Specifications for Wireless Personal Area Networks (WPANs) used in or 12 around a body," IEEE, 2011

[9] E. J. WELDON, JR., "An Improved Selective-Repeat ARQ Strategy," Communications, IEEE Transactions on, Volume: 30 , Issue: 3, pp. 480-486, Mar. 1982

[10] William E. Ryan, Shu Lin, "Channel Codes Classical and Modern," Cambridge, Cambridge University 
Press, 2009

[11] L. H. Charles Lee, "Convolutional Coding: Fundamentals and Applications," Boston, London, Artech House, 1997

[12] Kamya Yekeh Yazdandoost, Kamran Sayrafian-Pour," Channel Model for Body Area Network (BAN)," IEEE P802.15Working Group for Wireless Per-sonal Area Networks(WPANs), IEEE P802.15-08-0780-10-0006, April 2009

[13] Toyoo Takata, Yuji Yamashita, Toru Fujiwara, Tadao Kasami, and Shu Lin, "Suboptimum decoding of decomposable block codes," Information Theory, IEEE Transactions on, Issue: 5, pp.1392-1405, Sep 1994

[14] Hemmati F, "Closest coset decoding of $|u| u+v \mid$ codes", Selected Areas in Communications, IEEE Journal on, Volume : 7, Issue:6, pp. 982 - 988, Aug 1989

[15] Marc P.C. Fossorier, Shu Lin, "Some decomposable codes: the $|a+x| b+x|a+b+x|$ construction", Information Theory, IEEE Transactions on, Volume : 43, Issue:5, pp.1663 - 1667, Sep 1997

[16] Jari Iinatti, Matti Hämäläinen, Ryuji Kohno, "On the Recent and Future Topics of Wireless Communications in Healthcare",

JAPANESE-FINNISH JOINT SYMPOSIUM ICT and Technology in Medical and Health Research, Dec 2011

[17] Takuji Suzuki, Hirokazu Tanaka, Shigenobu Minami, Hiroshi Yamada, Takashi Miyata, "Wearable Wireless Vital Monitoring Technology for Smart Health Care", 2013 7th International Symposium on Medical Information and Communication Technology (ISMICT), Mar 2013 